\title{
Analysis of Genetic Variants in Myeloproliferative Neoplasms Using a 22-Gene Next-Generation Sequencing Panel
}

\author{
Jaymi Tan \\ Sunway University \\ Yock Ping Chow \\ Sunway Medical Centre \\ Norziha Zainul Abidin \\ Sunway Medical Centre \\ Kian Meng Chang \\ Sunway Medical Centre \\ Veena Selvaratnam \\ Ampang Hospital \\ Nor Rafeah Tumian \\ Universiti Kebangsaan Malaysia Medical Centre \\ Yang Ming Poh \\ Perdana University \\ Abhi Veerakumarasivam \\ Sunway University \\ Michael Arthur Laffan \\ Imperial College London \\ Chieh Lee Wong ( $\nabla$ wongchiehlee@hotmail.com ) \\ Sunway Medical Centre
}

\section{Research Article}

Keywords: myeloproliferative neoplasm, next-generation sequencing, gene, mutation, variant, bioinformatic, disease management

Posted Date: August 19th, 2021

DOI: https://doi.org/10.21203/rs.3.rs-721996/v1

License: @ (i) This work is licensed under a Creative Commons Attribution 4.0 International License. Read Full License

Version of Record: A version of this preprint was published at BMC Medical Genomics on January 15th, 2022. See the published version at https://doi.org/10.1186/s12920-021-01145-0. 


\section{Abstract \\ Background}

The Philadelphia (Ph)-negative myeloproliferative neoplasms (MPNs) (i.e. essential thrombocythaemia (ET), polycythaemia vera (PV), and primary myelofibrosis (PMF)) are a group of chronic clonal haematopoietic disorders that have the propensity to advance into bone marrow failure or acute myeloid leukaemia; often resulting in fatality. Although driver mutations have been identified in these Ph-negative MPNs, subtype-specific markers of the disease have yet to be discovered. Next-generation sequencing (NGS) technology can potentially improve the clinical management of Ph-negative MPNs by allowing for the simultaneous screening of many disease-associated genes.

\section{Methods}

The performance of a custom, in-house designed 22-gene NGS panel was technically validated using reference standards across two independent replicate runs. The panel was subsequently used to screen a total of 10 clinical MPN samples $(E T n=3, P V n=3, P M F n=4)$. The resulting NGS data was then analysed via a bioinformatics pipeline.

\section{Results}

The custom NGS panel had a detection limit of $1 \%$ variant allele frequency (VAF). A total of 44 variants with minor allele frequencies (MAFs) of $\geq 1 \%$, and 20 variants with MAFs of $<1 \%$ were identified ( 4 of which were putatively novel variants with potential biological significance) across the 10 MPN samples. All single nucleotide variants with VAFs $\geq 15 \%$ were confirmed via Sanger sequencing.

\section{Conclusions}

The high fidelity of the NGS analysis and the identification of known and novel variants support its potential clinical utility in the management of Ph-negative MPNs.

\section{Background}

Myeloproliferative neoplasms (MPNs) are a group of chronic clonal haematopoietic disorders. The MPN subtypes include the Philadelphia (Ph) chromosomepositive chronic myeloid leukaemia, as well as the Ph-negative MPNs such as essential thrombocythaemia (ET) (characterised by an overproduction of platelets), polycythaemia vera (PV) (characterised by an overproduction of red blood cells), and primary myelofibrosis (PMF) (characterised by progressive bone marrow fibrosis, and is further subdivided into an early, prefibrotic stage (pre-PMF) and a late, overt fibrotic stage (overt-PMF)). Among the three, ET is the most indolent, whereas PMF is associated with the highest symptom burden and worst prognosis. Common causes of morbidity and mortality include thromboembolic and/or haemorrhagic complications, as well as disease progression to myelofibrosis (MF) and/or transformation to acute myeloid leukaemia (AML), all of which vary in frequency between MPN subtypes [1]. To date, allogenic stem cell transplantation remains the only curative option for MPNs but it is often not considered due to age-related co-morbidities and high transplant-associated mortality rates. Hence, available treatment options such as phlebotomy, aspirin, hydroxyurea, anagrelide, pegylated interferons, and JAK inhibitors are primarily aimed at reducing the risk of disease complications, disease progression, and malignant transformation [2,3].

The management of MPNs require an integrated, multimodal approach that includes the evaluation of clinical features, bone marrow morphology, immunophenotype, cytogenetics, as well as molecular genetics, as recommended in the latest World Health Organisation (WHO) classification guidelines [4]. As MPNs are chronic in nature, cases are often asymptomatic upon diagnosis and discovered incidentally through physical examination or abnormal blood counts [4]. Typically, the diagnostic procedure includes molecular tests for MPN driver mutations in JAK2 (V617F or exon 12 mutations), CALR (exon 9 insertions and deletions) and MPL (W515 mutations), as well as the morphological analysis of the peripheral blood smear and bone marrow aspirate/trephine biopsy; the findings of which are correlated with the results of the full blood count. Therapeutic decisions are then made based on the patient's mutational landscape, disease burden, as well as disease prognosis. However, the MPN driver mutations in JAK2, CALR and MPL are not MPN subtype-specific, and can also be found in other myeloid diseases such as myelodysplastic syndromes (MDSs), myelodysplastic/myeloproliferative neoplasms (MDS/MPNs) and AML $[4,5]$. In addition, there are also MPN cases that are triple-negative (TN) for any of the driver mutations. Morphological features such as the degree of bone marrow fibrosis, used for disease diagnosis, are subjective in nature and thus have high inter-observer variability [6-8]. This complicates accurate diagnosis as the subtypes such as ET and pre-PMF share similar presenting features of marked thrombocytosis but are different in disease progression risk and outcomes $[4,8]$.

The significant genotypic and phenotypic heterogeneity that exist between MPN subtypes and other myeloid disorders remains a challenge towards effective management of MPNs. Rapid advancements in gene sequencing technology in the last decade have led to the discovery of other MPN-associated genetic aberrations, such as mutations in ASXL1, EZH2, TET2, IDH1, IDH2, SRSF2 and SF3B1, contributing towards improved disease prognostication. In a novel prognostic model developed by Grinfeld, et al. [9], patients with MPN were stratified into eight groups by combining genomic data with traditional laboratory and clinical findings. Compared to current prognostic models, the model was superior in performance and was able to better define disease outcomes, especially within the "intermediate-risk" categories of the current prognostic models [9]. Nevertheless, further validation studies and comprehensive genetic profiling, i.e. through next-generation sequencing (NGS) technology, is required for such a model to be implemented in clinic worldwide. Here, we report on the validation of a custom NGS panel which targets $22 \mathrm{MPN}$-associated genes (ABL1, ASXL1, CALR, CBL, CEBPA, CSF3R, DNMT3A, EZH2, FLT3, IDH1, IDH2, JAK2, 
KIT, MPL, NPM1, PDGFRA, RUNX1, SF3B1, SRSF2, TET2, TP53, and U2AF1). Using a set of reference standards and a small cohort of clinical MPN samples, we demonstrated that the custom NGS panel performed robustly and enabled the detection of known MPN-associated genetic variants, as well as previously unreported variants of potential biological significance. The findings not only support the clinical utility of the custom NGS panel, but also contribute to the MPN knowledge base through the identification of novel variants.

\section{Materials And Methods}

\subsection{Ethics Statement}

A multicentre study was conducted across three institutions in Malaysia, namely Sunway Medical Centre, Ampang Hospital, and Universiti Kebangsaan Malaysia Medical Centre. Ethics approval for this study was obtained from the Sunway Medical Centre Independent Research Ethics Committee (SREC 008/2018/FR), Universiti Kebangsaan Malaysia Medical Centre Research Ethics Committee (UKM FPR.4/244/FF-2018-420), Medical Research \& Ethics Committee Ministry of Health Malaysia (MREC 8KKM/NIHSEC/P-19-99), and Sunway University Research Ethics Committee (PGSUREC 2019/010).

\subsection{Custom NGS Panel}

An Ampliseq for Illumina custom NGS panel was designed using the Illumina Design Studio tool (Illumina, San Diego, USA) to target the hotspot exons of 22 genes known to be frequently mutated in MPNs, namely ABL1, ASXL1, CALR, CBL, CEBPA, CSF3R, DNMT3A, EZH2, FLT3, IDH1, IDH2, JAK2, KIT, MPL, NPM1, PDGFRA, RUNX1, SF3B1, SRSF2, TET2, TP53, and U2AF1 (Supplementary Table S1 and S2).

\subsection{Technical Validation of Custom NGS Panel Performance}

The custom NGS panel performance was validated using four reference standards through two identical but independent NGS runs (Supplementary Table S3). The reference standards used were: 1) Tru-Q0 (100\% wild-type) Reference Standard (Horizon, Cambridge, UK) - as a negative control; 2) Tru-Q1 (5\% Tier) Reference Standard (Horizon) - as a positive control for 5\% mutant allele in FLT3 (p.Al836), IDH1 (p.R132C), and JAK2 (p.V617F); 3) Tru-Q7 (1.3\% Tier) Reference Standard (Horizon) - as a positive control for 1.3\% mutant allele in FLT3 (p.D835Y, p.AI836), IDH1 (p.R132C/H), IDH2 (p.R140Q, p.R172K), JAK2 (p.V617F), KIT (p.D816V), and PDGFRA (p.D842V); and 4) Seraseq ${ }^{\text {TM }}$ Myeloid Mutation DNA Mix (SeraCare, Milford, USA) - as a positive control for mutant allele in $A B L 1$ (p.T315l), ASXL1 (p.E635fs*15, p.G646fs*12), CALR (p.L367fs*46), CBL (p.R420Q, p.L380P), CEBPA (p.H24fs*84, p.K313_V314insK), CSF3R (p.T618I), FLT3 (c.1759_1800dup, duplication of chr13:28,608,250 - 28,608,277, p.D835Y), IDH1 (p.R132C), JAK2 (p.V617F, p.N542_E543del), MPL (p.W515L), NPM1 (p.W288fs*12), SF3B1 (p.K700E, p.K666N), SRSF2 (p.P95_R102del), and U2AF1 (p.S34F) (Supplementary Table S3). The NGS assay performance was assessed based on the sensitivity, specificity, repeatability, concordance, and positive predictive values.

\subsection{Study Cohort}

Ten patients who were diagnosed with ET, PV or PMF according to the revised 4th edition of the WHO classification guidelines [4] in the participating institutions were recruited for this study. All patients who agreed to participate in the study provided their written consent. Approximately $10 \mathrm{~mL}$ peripheral blood samples from each patient were collected in EDTA tubes and processed within 24 hours to extract the DNA from the cells. Patient demographic and clinical data were recorded by using standardised forms.

\subsection{Genomic DNA Extraction and Quality Control}

Genomic DNA was extracted from each blood sample using the Maxwell $\circledast$ RSC Whole Blood DNA Kit (Promega, Madison, USA) according to the manufacturer's guidelines. The purity $\left(A_{260} / A_{280}\right.$ and $\left.A_{260} / A_{230}\right)$ and concentration of extracted DNA was assessed using the Nanodrop ${ }^{\text {TM }}$ (Thermo Fisher Scientific, Waltham, USA) spectrophotometer. DNA samples with satisfactory purity ratios of $1.80-2.00\left(A_{260} / A_{280}\right)$ and $2.00-2.20\left(A_{260} / A_{230}\right)$ were visualised using $1 \%$ agarose gel and kept for downstream experiments.

\subsection{Preparation of NGS Libraries and Sequencing}

The NGS libraries were prepared according to the AmpliSeq for Illumina On-Demand, Custom and Community Panels Reference Guide Protocol, DNA Panels Standard Workflow Procedure for Three Primer Pools, using reagents from the AmpliSeq ${ }^{\text {TM }}$ Library PLUS for Illumina ${ }^{\circledR}$. The concentration of the input DNA was measured using the Qubit dsDNA BR Assay and the concentration of the constructed libraries was assessed using the Qubit dsDNA HS Assay on the Qubit 2.0 Fluorometer (Thermo Fisher Scientific) following the manufacturer's protocol. The quality of the constructed libraries was assessed using the DNA Hi Sens Lab Chip (PerkinElmer, Waltham, USA) according to the DNA High Sensitivity Assay User Guide for LabChip GX Touch/GXII Touch Standard Sample Workflow. Libraries were denatured and diluted according to the MiSeq System Denature and Dilute Libraries Guide Protocol A: Standard Normalization Method for MiSeq Reagent Kit v2, with a PhiX library used as a sequencing control. Pooled sequencing of 6 samples per run was conducted on an Illumina Miseq using the MiSeq Reagent Micro Kit v2 ( 2 x 150 base pair (bp), paired end) to a minimum coverage depth of 5000x according to the manufacturer's protocol.

\subsection{Quality Control and Bioinformatics Analysis of NGS data}

The sequencing metrics were visualized using the Illumina Sequence Analysis Viewer software. The quality of the raw NGS data was assessed using the FastQC software on the Illumina BaseSpace ${ }^{\mathrm{Tm}}$ Sequence Hub. The sequencing data was analysed using a combination of two sequence alignment and variant calling applications (apps) also on the Illumina BaseSpace ${ }^{\text {TM }}$ Sequence Hub - the DNA Amplicon app and Pindel app, at a $1 \%$ somatic variant allele frequency (VAF) detection limit. The DNA Amplicon app is designed to detect small variants, whereas the Pindel app is designed to detect larger structural variants such as large deletions (as large as 10kb), medium sized insertions, inversions and tandem duplications [10]. The DNA Amplicon app also generates a detailed report that summarises various information including sample read quality, amplicon data, base level statistics, and coverage by amplicon region. The called variants were then annotated using wANNOVAR (Wang Genomics Lab, Philadelphia, USA). 
For the technical validation of the custom NGS panel, only variants that were present in the four reference standards were filtered-in and prioritized; whereas for the screening of the clinical MPN samples, all annotated variants were manually reviewed by filtering and prioritizing using the following criteria: 1 ) all variants except for exonic variants were excluded, 2) variants with minor allele frequencies (MAFs) of $\geq 1 \%$ (as reported in the 1000 Genomes Project, ExAC, ESP6500, and gnomAD databases) were excluded, and 3) potential sequencing errors (variants with a VAF of $<5 \%$ and appear in all samples) were excluded. Aligned read (.bam) files for all samples were then manually inspected to confirm the presence of the filtered-in and prioritized variants using the Integrative Genomics Viewer (IGV) software (Broad Institute, Cambridge, USA). The confirmed variants were manually checked against the ClinVar [11], COSMIC [12], and dbSNP [13] databases and those that were not found in either one of those databases were considered as putative novel variants.

\subsection{Variant confirmation via Sanger Sequencing}

The Ensembl genome browser [14] and NCBI Primer-BLAST [15] were used to design PCR primers for the target variants (Supplementary Table S4). Each PCR mixture contained $17 \mu \mathrm{L}$ of nuclease free water, $25 \mu \mathrm{L}$ of the Amplitaq Gold® 360 Master Mix (Thermo Fisher Scientific), $2 \mu \mathrm{L} 360 \mathrm{GC}$ Enhancer (Thermo Fisher Scientific), $2 \mu \mathrm{L}$ each of $5 \mu \mathrm{M}$ forward and reverse primer, and $2 \mu \mathrm{L}$ of $50 \mathrm{ng} / \mu \mathrm{L}$ sample DNA. For the TET2 exon 7 p.D1314Mfs*48 variant, the $360 \mathrm{GC}$ Enhancer in the PCR mixture was found to reduce the PCR product yield, and was therefore replaced with nuclease free water. The PCR thermocycling conditions for all NGS detected variants were as follows: $95^{\circ} \mathrm{C}$ for $10 \mathrm{~min}, 35$ cycles at $95^{\circ} \mathrm{C}$ for $30 \mathrm{~s}, 55^{\circ} \mathrm{C}$ for $30 \mathrm{~s}$, and $72^{\circ} \mathrm{C}$ for $1 \mathrm{~min}$, followed by a final extension at $72^{\circ} \mathrm{C}$ for $7 \mathrm{~min}$. The PCR products were visualised using a $1 \%$ agarose gel to confirm successful amplification and then purified using Wizard $\circledast$ SV Gel and PCR Clean-Up System (Promega) by centrifugation according to the manufacturer's instructions but eluted in $15 \mathrm{~mL}$ of nuclease free water twice to

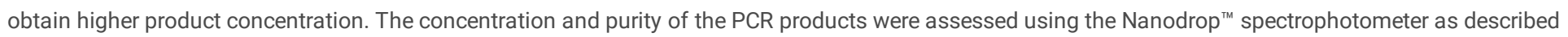
in Sect. 2.5. Samples with satisfactory concentration and purity ratios were sent for Sanger sequencing. Alignment of Sanger sequence (.seq) files were conducted via the ClustalW algorithm using the Jalview software [16] whereas the Sanger sequence chromatograms were analyzed using the 4peaks software (Nucleobytes, Aalsmeer, Netherlands). The pathogenicity of the variants was assessed using ClinVar [11].

\section{Results}

\subsection{Technical validation of the custom NGS panel}

The custom NGS panel performance was evaluated using a set of reference standards in two identical but independent NGS runs. After library preparation, all samples were confirmed to be of sufficient quality and quantity. The majority of the constructed libraries were within the targeted size range of 400 bp for sequencing using the custom NGS panel (Supplementary Figure S1). Both NGS runs achieved cluster densities of 860 to $878 \mathrm{~K} / \mathrm{mm}^{2}$, $>93 \%$ of clusters passed the quality filter, and $>95 \%$ of the read bases with quality scores of above Q30, which were close to The Miseq System specifications of $865-965 \mathrm{~K} / \mathrm{mm} 2$ cluster density and $>80 \%$ of bases above Q30 (Supplementary Table S5). Each sample achieved $~ 99.5 \%$ on-target aligned reads, and minimum amplicon mean coverage depths of $4600 x$ (Supplementary Table S6). Analysis of the coverage depth per amplicon region revealed that $98.6 \%$ of the targeted regions ( $\mathrm{n}$ $=216 / 219$ amplicons) had average coverage depths of $>1000 x$. Two amplicons had average coverage depths of < 1000x, namely AMPL89337 (DNMT3A exon 17, chr2:25464411-25464625) and AMPL1156 (TP53 exon 4/exon 5, chr17:7578360-7578579), while AMPL117202 (MPL exon 10, chr1:4381490243815103) had a coverage depth of below 100x (Supplementary Figure S2).

Combined analysis of sequencing results with the DNA Amplicon and Pindel apps revealed that the former was able to detect all known variants in the reference standards except for large duplications in FLT3; whereas Pindel was able to detect all frameshift variants as well as large duplications in FLT3, but not single nucleotide variants (SNVs) (Fig. 1). Overall, the custom NGS panel has a sensitivity of $99.2 \%$, a specificity of $96.3 \%$, a positive predictive value of $97.7 \%$, an average intra-run concordance of $98.8 \%$ [range $95.2-100 \%$ ], an average inter-run concordance of $99.0 \%$ [range $95.2-100 \%$ ], and a detection limit of $1 \%$ VAF (Fig. 1).

\subsection{Study Cohort Demographics and Clinical Data}

A total of 10 MPN patients (ET $n=3, P V n=3$, PMF $n=4)$ were recruited for this study (Sunway Medical Centre $(n=7)$, Ampang Hospital $(n=1)$ and Universiti Kebangsaan Malaysia Medical Centre $(n=2)$ ) (Table 1). All patients were diagnosed based on the latest WHO criteria [4]. The median age of diagnosis was 52 years (range: 30 to 79 years). The majority of the patients were male $(n=7 / 10)$. Half of the study cohort was of Chinese ethnicity, 4 were Malay, and 1 was Dutch. Six patients presented with constitutional symptoms, and only 1 patient (Sample 09, overt-PMF) presented with hepatosplenomegaly. Half of the study cohort had a history of smoking. Seven patients were tested positive for the JAK2 V167F driver mutation, and 3 were positive for CALR driver mutations. No patients tested positive for MPL driver mutations. 
Table 1

Study Sample demographics and clinical data.

\begin{tabular}{|c|c|c|c|c|c|c|c|c|c|c|c|}
\hline Sample & $\begin{array}{l}\text { MPN } \\
\text { subtype }\end{array}$ & $\begin{array}{l}\text { Age } \\
\text { (Years) }\end{array}$ & Sex & Ethnicity & $\begin{array}{l}\mathrm{Hb} \\
(\mathrm{g} / \mathrm{dL})\end{array}$ & $\begin{array}{l}\text { WBC } \\
\left(\times 10^{9} / L\right)\end{array}$ & $\begin{array}{l}\text { Platelet } \\
\left(\times 10^{9} / \mathrm{L}\right)\end{array}$ & HSM & $\begin{array}{l}\text { Constitutional } \\
\text { symptoms }\end{array}$ & Smoker & $\begin{array}{l}\text { NGS-detected } \\
\text { variants (VAF\%) }\end{array}$ \\
\hline \multirow[t]{3}{*}{01} & ET & 30 & M & Malay & 17.1 & 7.6 & 1730 & No & No & No & $\begin{array}{l}\text { CALR K385Ifs*46 } \\
(35.2 \%)\end{array}$ \\
\hline & & & & & & & & & & & $\begin{array}{l}\text { CALR K385T } \\
(35.5 \%)\end{array}$ \\
\hline & & & & & & & & & & & $\begin{array}{l}\text { TET2 I1195V } \\
(49.6 \%)\end{array}$ \\
\hline \multirow[t]{3}{*}{02} & ET & 50 & $\mathrm{~F}$ & Chinese & 13.2 & 10.9 & 776 & No & No & No & $\begin{array}{l}\text { CALR K385Nfs*46 } \\
(15.9 \%)\end{array}$ \\
\hline & & & & & & & & & & & $\begin{array}{l}\text { TET2 E1513G } \\
(51.6 \%)\end{array}$ \\
\hline & & & & & & & & & & & $\begin{array}{l}\text { TET2 F868L } \\
(48.7 \%)\end{array}$ \\
\hline \multirow[t]{3}{*}{03} & ET & 79 & M & Malay & 11.1 & 24.5 & 869 & No & No & Yes & $\begin{array}{l}J A K 2 \text { V } 617 \mathrm{~F} \\
(88.0 \%)\end{array}$ \\
\hline & & & & & & & & & & & $\begin{array}{l}\text { ASXL1 Q1433Q } \\
(49.2 \%)^{\dagger}\end{array}$ \\
\hline & & & & & & & & & & & $\begin{array}{l}\text { DNMT3A P385P } \\
(51.6 \%)\end{array}$ \\
\hline \multirow[t]{2}{*}{04} & PV & 54 & M & Chinese & 18.9 & 17.2 & 426 & No & Yes & Yes & $\begin{array}{l}J A K 2 \text { V } 617 \mathrm{~F} \\
(72.9 \%)\end{array}$ \\
\hline & & & & & & & & & & & $\begin{array}{l}\text { ASXL1 } \\
\text { G646Wfs*10 } \\
(31.3 \%)^{\dagger}\end{array}$ \\
\hline 05 & PV & 66 & $\mathrm{~F}$ & Chinese & 19.3 & 18.1 & 423 & No & Yes & No & $\begin{array}{l}J A K 2 \text { V617F } \\
(66.5 \%)\end{array}$ \\
\hline \multirow[t]{2}{*}{06} & PV & 73 & M & Dutch & 20.0 & 9.0 & 334 & No & Yes & Yes & $\begin{array}{l}\text { JAK2 V617F } \\
(19.2 \%)\end{array}$ \\
\hline & & & & & & & & & & & $\begin{array}{l}\text { U2AF1 Q157P } \\
(9.8 \%)\end{array}$ \\
\hline \multirow[t]{2}{*}{07} & $\begin{array}{l}\text { Pre- } \\
\text { PMF }\end{array}$ & 50 & M & Chinese & 15.0 & 17.8 & 859 & No & Yes & Yes & $\begin{array}{l}\text { JAK2 V617F } \\
(12.1 \%)\end{array}$ \\
\hline & & & & & & & & & & & $\begin{array}{l}A B L 1 \mathrm{~N} 331 \mathrm{~S} \\
(48.8 \%)\end{array}$ \\
\hline \multirow[t]{5}{*}{08} & $\begin{array}{l}\text { Pre- } \\
\text { PMF }\end{array}$ & 50 & $F$ & Malay & 8.7 & 58.4 & 1099 & No & Yes & No & $\begin{array}{l}\text { JAK2 V617F } \\
(53.7 \%)\end{array}$ \\
\hline & & & & & & & & & & & $\begin{array}{l}\text { RUNX1 Q308H } \\
(49.6 \%)\end{array}$ \\
\hline & & & & & & & & & & & $\begin{array}{l}\text { SF3B1 K700E } \\
(45.6 \%)\end{array}$ \\
\hline & & & & & & & & & & & $\begin{array}{l}\text { TET2 } \\
\text { D1314Mfs*48 } \\
(46.3 \%)\end{array}$ \\
\hline & & & & & & & & & & & $\begin{array}{l}\text { TET2Y1245C } \\
(44.9 \%)^{\dagger}\end{array}$ \\
\hline \multirow[t]{2}{*}{09} & $\begin{array}{l}\text { Overt- } \\
\text { PMF }\end{array}$ & 46 & M & Malay & 7.1 & 3.9 & 452 & Yes & No & No & $\begin{array}{l}\text { CALRL367Tfs*45 } \\
(10.6 \%)\end{array}$ \\
\hline & & & & & & & & & & & $\begin{array}{l}\text { ASXL } 1 \mathrm{~L} 731 \mathrm{Yfs}^{*} 12 \\
(38.5 \%)^{\dagger}\end{array}$ \\
\hline \multirow[t]{2}{*}{10} & $\begin{array}{l}\text { Overt- } \\
\text { PMF }\end{array}$ & 63 & M & Chinese & 7.3 & 5.5 & 54 & No & Yes & Yes & $\begin{array}{l}\text { JAK2 V617F } \\
(12.2 \%)\end{array}$ \\
\hline & & & & & & & & & & & $\begin{array}{l}\text { ASXL } 1 \text { Y } 591 \mathrm{fs} * 0 \\
(40.8 \%)\end{array}$ \\
\hline
\end{tabular}

${ }^{\dagger}$ Putative novel variant. Hb, Haemoglobin; WBC, White blood cell; HSM, Hepatosplenomegaly. 


\begin{tabular}{|c|c|c|c|c|c|c|c|c|c|c|c|}
\hline Sample & $\begin{array}{l}\text { MPN } \\
\text { subtype }\end{array}$ & $\begin{array}{l}\text { Age } \\
\text { (Years) }\end{array}$ & Sex & Ethnicity & $\begin{array}{l}\mathrm{Hb} \\
(\mathrm{g} / \mathrm{dL})\end{array}$ & $\begin{array}{l}\text { WBC } \\
\left(\times 10^{9} / L\right)\end{array}$ & $\begin{array}{l}\text { Platelet } \\
\left(\times 10^{9} / \mathrm{L}\right)\end{array}$ & HSM & $\begin{array}{l}\text { Constitutional } \\
\text { symptoms }\end{array}$ & Smoker & $\begin{array}{l}\text { NGS-detected } \\
\text { variants (VAF\%) }\end{array}$ \\
\hline & & & & & & & & & & & $\begin{array}{l}\text { TET2 A304V } \\
(50.1 \%)\end{array}$ \\
\hline & & & & & & & & & & & $\begin{array}{l}\text { U2AF1 Q157P } \\
(42.5 \%)\end{array}$ \\
\hline
\end{tabular}

\subsection{Identification of Genetic Variants in Clinical MPN Samples}

An initial total of 314 unique variants were detected across all 10 clinical MPN samples (Fig. 2). After filtering out intronic and UTR variants as well as all variants with MAFs of $\geq 1 \%$, there were 115 exonic variants; 95 of which were found to be sequencing errors and were subsequently excluded. A total of 20 unique variants across the 10 clinical MPN samples were identified, including known MPN driver mutations (Table 2). 
Table 2

Details of all NGS detected variants across the 10 clinical MPN samples.

\begin{tabular}{|c|c|c|c|c|c|c|c|c|}
\hline Gene & c.DNA & Aa change & Consequence & dbSNP & $\begin{array}{l}\text { ClinVar Assertion/COSMIC } \\
\text { ID }\end{array}$ & Sample & $\begin{array}{l}\text { VAF } \\
\text { (\%) }\end{array}$ & San! \\
\hline CALR & c.1092_1143del & p.L367Tfs*45 & Fs del & NA & Pathogenic/COSM1738055 & $\begin{array}{l}\text { 09, } \\
\text { Overt- } \\
\text { PMF }\end{array}$ & 10.6 & $\checkmark$ \\
\hline CALR & c.1154_1155insTTGTC & p.K385Nfs*46 & Fs ins & rs765476509 & CosM1738056 & $02, \mathrm{ET}$ & 15.9 & $\checkmark$ \\
\hline CALR & c.1153_1154insTATGT & p.K385lfs*46 & Fs ins & NA & cosM5985669 & 01, ET & 35.2 & $\checkmark$ \\
\hline CALR & c.A1154C & p.K385T & nSNV & rs 1024435400 & NA & 01, ET & 35.5 & $\checkmark$ \\
\hline \multirow[t]{7}{*}{ JAK2 } & c.G1849T & p.V617F & nSNV & rs77375493 & Pathogenic/COSM12600 & $\begin{array}{l}\text { 07, Pre- } \\
\text { PMF }\end{array}$ & 12.1 & $x$ \\
\hline & & & & & & $\begin{array}{l}10, \\
\text { Overt- } \\
\text { PMF }\end{array}$ & 12.2 & $x$ \\
\hline & & & & & & $06, \mathrm{PV}$ & 19.2 & $\checkmark$ \\
\hline & & & & & & $\begin{array}{l}\text { 08, Pre- } \\
\text { PMF }\end{array}$ & 53.7 & $\checkmark$ \\
\hline & & & & & & $05, P V$ & 66.5 & $\checkmark$ \\
\hline & & & & & & $04, P V$ & 72.9 & $\checkmark$ \\
\hline & & & & & & 03, ET & 88.0 & $\checkmark$ \\
\hline ABL1 & c.A1049G & p.N350S & nSNV & rs144448357 & NA & $\begin{array}{l}\text { 07, Pre- } \\
\text { PMF }\end{array}$ & 48.8 & $\checkmark$ \\
\hline \multirow[t]{4}{*}{ ASXL1 } & c.1927_1928insGGGGGGGGTG & p.G646Wfs* $10^{\dagger}$ & stopgain & rs750318549 & NA & $04, P V$ & 31.3 & $\checkmark$ \\
\hline & GCCCGGGTGGAGGTGGCGG & & & & & & & \\
\hline & CGGGGCCACCGATGAGGGG & & & & & & & \\
\hline & GGGGGCAGAGGCAGCAGCA & & & & & & & \\
\hline ASXL1 & c.1772dupA & p.Y591fs*0 & stopgain & rs762036456 & $\begin{array}{l}\text { COSM4169775, } \\
\text { COSM4169776 }\end{array}$ & $\begin{array}{l}10, \\
\text { Overt- } \\
\text { PMF }\end{array}$ & 40.8 & $\checkmark$ \\
\hline ASXL1 & c.2190delC & p.L731Yfs* ${ }^{\star} 12^{\dagger}$ & Fs del & NA & NA & $\begin{array}{l}\text { 09, } \\
\text { Overt- } \\
\text { PMF }\end{array}$ & 38.5 & $\checkmark$ \\
\hline ASXL1 & c.A4299G & p.Q1433Q ${ }^{\dagger}$ & sSNV & NA & NA & 03, ET & 49.2 & $\checkmark$ \\
\hline DNMT3A & c.G1155A & p.P385P & sSNV & rs368009374 & VUS/likely benign & 03, ET & 51.6 & NA \\
\hline RUNX1 & c.G924T & p.Q308H & nSNV & rs80314254 & Benign & $\begin{array}{l}\text { 08, Pre- } \\
\text { PMF }\end{array}$ & 49.6 & $\checkmark$ \\
\hline SF3B1 & c.A2098G & p.K700E & nSNV & rs559063155 & $\begin{array}{l}\text { Likely pathogenic/ } \\
\text { COSM } 84677\end{array}$ & $\begin{array}{l}\text { 08, Pre- } \\
\text { PMF }\end{array}$ & 45.6 & $\checkmark$ \\
\hline TET2 & c.C911T & p.A304V & nSNV & NA & $\begin{array}{l}\text { COSM5610834, } \\
\text { COSM5610835 }\end{array}$ & $\begin{array}{l}10, \\
\text { Overt- } \\
\text { PMF }\end{array}$ & 50.1 & $\checkmark$ \\
\hline TET2 & c.T2604G & p.F868L & nSNV & rs147836249 & CosM87107 & $02, \mathrm{ET}$ & 48.7 & $\checkmark$ \\
\hline TET2 & c.A3583G & p.I1195V & nSNV & rs568009712 & NA & 01, ET & 49.6 & $\checkmark$ \\
\hline TET2 & c.A3734G & p.Y1245C ${ }^{\dagger}$ & nSNV & NA & NA & $\begin{array}{l}\text { 08, Pre- } \\
\text { PMF }\end{array}$ & 44.9 & $\checkmark$ \\
\hline TET2 & c.3937delG & p.D1314Mfs*48 & Fs del & NA & COSM4383928 & $\begin{array}{l}\text { 08, Pre- } \\
\text { PMF }\end{array}$ & 46.3 & $\checkmark$ \\
\hline TET2 & c.A4538G & p.E1513G & nSNV & rs553669299 & NA & $02, \mathrm{ET}$ & 51.6 & $\checkmark$ \\
\hline \multirow[t]{2}{*}{ U2AF1 } & c.A470C & p.Q157P & nSNV & rs371246226 & Likely pathogenic/ & $06, \mathrm{PV}$ & 9.8 & $x$ \\
\hline & & & & rs371246226 & $\begin{array}{l}\text { COSM211534, } \\
\text { CosM1318797 }\end{array}$ & $\begin{array}{l}10, \\
\text { Overt- } \\
\text { PMF }\end{array}$ & 42.5 & $\checkmark$ \\
\hline
\end{tabular}

${ }^{\dagger}$ Putative novel variant; Aa change, Amino acid change; Fs del, Frameshift deletion; Fs ins, Frameshift insertion; $\checkmark$, Sanger detected; $X$, Sanger undetected; $N$ Data not available. 
On average, the PMF samples appeared to harbour the highest number of variants, whereas the PV samples appeared to harbour the least number of variants (Fig. 3). Among the 20 variants, 13 were SNVs (synonymous SNV (sSNV) $n=2$, nonsynonymous SNV (nSNV) $n=11$ ) and 7 were indels (frameshift insertion (fs ins), $\mathrm{n}=2$; frameshift deletions (fs del), $\mathrm{n}=3$; stopgain, $\mathrm{n}=2$ ). Out of the 10 sequenced clinical samples, the JAK2 V617F driver mutation was identified in 7 samples, while $C A L R$ driver mutation was identified in 3 samples. Aside from driver mutations, other variants were also detected, including an nSNV in $C A L R$ as well as variants in $A B L 1(\mathrm{n}=1), A S X L 1(\mathrm{n}=4), \operatorname{DNMT3A}(\mathrm{n}=1)$; RUNX1 $(\mathrm{n}=1), \operatorname{SF} 3 B 1(\mathrm{n}=1), \operatorname{TET} 2(\mathrm{n}=6)$, and U2AF1 ( $=2)($ Fig. 3). All NGS-detected variants with allele frequencies of $\geq 15 \%$ were confirmed via Sanger sequencing (Table 2), except for the DNMT3A P385P sSNV that was not confirmed due to nonspecific amplification (Supplementary Figure S3).

The $A B L 1$ point mutation identified in this study, N331S (Sample 07, pre-PMF) was reported in dbSNP (rs144448357). However, it is unknown whether it was previously identified in MPN due to the lack of ClinVar and COSMIC data (Table 2). The variant was identified in a patient diagnosed with pre-PMF at 50 years of age, with JAK2 V617F mutation. The patient presented with higher-than-normal platelet $\left(859 \times 10^{9} / \mathrm{L}\right)$ and white cell counts $\left(17.8 \times 10^{9} / \mathrm{L}\right)$, as well as constitutional symptoms (Table 1).

Four ASXL 1 variants were identified in this study, of which, the $A S X L 1 \mathrm{G} 646 \mathrm{Wfs}{ }^{*} 10$

(c.1927_1928insGGGGGGGGTGGCCCGGGTGGAGGTGGCGGCGGGGCCACCGATGAGGGGGGGGGCAGAGGCAGCAGCA) stopgain variant (Sample 04, PV) was found to be located in the same dbSNP cluster rs750318549 as the previously reported ASXL1 Gly646Trpfs*12 (c.1934dupG) stopgain, which is the most common ASXL 1 mutation accounting for $>50 \%$ of all identified ASXL 1 mutations in myeloid malignancies [17] (Table 2). While ASXL 1 Gly646Trpfs ${ }^{\star} 12$ is the result of a duplication of a $\mathrm{G}$ nucleotide within a homopolymer region of eight $\mathrm{G}$ nucleotides [17], the variant $A S X L 1 \mathrm{G} 646 \mathrm{Wfs}{ }^{\star} 10$ identified in this study is the result of an insertion of 67 nucleotides at position chr20: 31022442, making it a novel stopgain variant. Two other ASXL 1 variants identified in this study, ASXL 1 L731Yfs*12 (Sample 09, overt-PMF) and Q1433Q (Sample 03, ET) were also putative novel variants with no dbSNP, COSMIC or ClinVar data; whereas the ASXL1 Y591fs*0 variant (Sample 10, overt-PMF) has been reported in various diseases including ET and MF [9, 18], MDS [19], chronic myelomonocytic leukaemia [20], AML [21], mast cell neoplasm [22] and CNL [23], as well as breast cancer [24], but has not been previously reported in PV (Table 2).

Six TET2 variants were identified in this study, of which, TET2 Y1245C (Sample 08, pre-PMF) was not found to be reported in dbSNP, COSMIC or the ClinVar database. The TET2 A304V (Sample 10, overt-PMF) and F868L (Sample 02, ET) variants have not been previously reported in MPN. TET2 A304V has only been previously identified in melanoma [25], while TET2 F868L has been previously identified in estrogen- and progesterone-receptor positive breast cancer [26], adult T cell lymphoma/leukaemia [27], and MDS [28, 29]. The TET2 D1314Mfs*48 variant (Sample 08, pre-PMF) has been reported in ET [30] and MDS [19]. Two other variants were reported in dbSNP - TET2 I1195V (rs568009712) (Sample 01, ET) and E1513G (rs553669299) (Sample 02, ET), but the associated disease(s) are unknown.

Two of the most common mutations in SF3B1 and U2AF1 were identified in this study, namely U2AF1 Q157P (rs371246226, COSM211534, COSM1318797) and SF3B1 K700E (rs559063155, COSM84677) [31-33]. Both variants have been reported to be likely pathogenic in the ClinVar database. A RUNX1 variant (Q308H rs80314254) reported as benign in familial thrombocytopenia in the ClinVar database was also identified. The variants SF3B1 K700E and RUNX1 Q308H were found in a pre-PMF sample (Sample 08) which also harboured the TET2 Y1245C and TET2 D1314Mfs*48 variants, alongside the JAK2 V617F driver mutation. The patient was 50 years of age and presented with abnormally high platelet count $\left(1099 \times 10^{\wedge} 9 / \mathrm{L}\right)$, anaemia $(\mathrm{Hb}=8.7 \mathrm{~g} / \mathrm{dL})$, and constitutional symptoms (Table 1). The U2AF1 Q157P variant was identified in PV and overt-PMF (Sample 06 and Sample 10, respectively) (Table 2). The overt-PMF sample (Sample 10) also harboured the ASXL 1 Y $591 \mathrm{fs} \star^{\star} 0$ variant in addition to the JAK2 V617F driver mutation. The patient was 63 years of age and presented with severe anaemia $(\mathrm{Hb}=7.3 \mathrm{~g} / \mathrm{dL})$, neutropenia $\left(\mathrm{WBC}=5.5 \times 10^{9} / \mathrm{L}\right)$ and thrombocytopenia $\left(\right.$ Platelet $\left.=54 \times 10^{9} / \mathrm{L}\right)$, with constitutional symptoms (Table 1).

\section{Discussion}

In this study, we evaluated the performance of our custom 22-gene NGS panel using reference standards and subsequently, a small cohort of clinical MPN samples. The 22 genes selected in this panel are known to be frequently mutated in myeloid neoplasms (not necessarily MPNs), and are known disease markers with diagnostic, prognostic and/or therapeutic value. First, the performance of the custom NGS panel was technically validated using reference standards in two identical but independent sequencing runs. The combined analysis of variants detected by the DNA Amplicon and Pindel tools revealed that the panel achieved high sensitivity, specificity, concordance and positive predictive values. The overall good performance of the panel and was further supported in its ability to detect variants with VAF values as low as 1\% (Fig. 1). The average depth-of-coverage per site across the panel was $5000 \mathrm{x}$, (Supplementary Figure S2). However, the lower average depth-of-coverage of the MPL amplicon (AMPL117202, MPL exon 10, chr1:43814902-43815103) at < $100 x$ could potentially lead to false negative results. In order for the panel to be used in the diagnosis of MPNs, optimisation of the sequencing coverage for the MPL region is critical for the detection of driver mutations in MPL. In addition, AMPL89337 (DNMT3A exon 17, chr2:25464411-25464625), AMPL1156 (TP53 exon 4/exon 5 chr17:7578360-7578579) and AMPL90417 (ASXL1 exon 13, chr20:31022935-31023187) were found to have average depths-ofcoverage of $<1000 x$ (Supplementary Figure S2, Additional File 1).

Uneven coverage or coverage bias may originate from various steps in the NGS workflow. The use of the PCR method during NGS library preparation has been found to be the primary contributor [34-36]. In PCR, the annealing efficiency of primers is commonly hindered by templates that are GC-rich that tend to remain double-stranded, as well as templates that are AT-rich (or GC-poor) that anneal poorly to primers. As a result, GC-and AT-rich regions (also known as low-complexity regions) are often poorly amplified and manifest in NGS as regions with low sequencing coverage. Further analysis of AMPL117202 showed that it had a GC content of $61 \%$ and AT content of $39 \%$, and a plot of the nucleotide distribution revealed that the GC content was $>60 \%$ in the majority of 30 bp windows (Supplementary Figure S4). Hence, it is likely that the majority of the AMPL117202 amplicons remained annealed as double-stranded DNA during PCR, leading to inefficient amplification and subsequent low NGS coverage of the amplicons. Future studies should involve the review of targeted regions and optimisation of NGS library preparation to improve the coverage of GC-rich regions, i.e. optimising PCR amplification conditions by using lower primer- 
extension temperature [34]. In order to rule out false negatives due to coverage bias, variants located in DNMT3A exon 17 and TP53 exon $4 /$ exon 5 should also be used to validate the performance of the custom panel.

From the screening of clinical MPN samples with the custom NGS panel, 20 unique variants including MPN driver mutations in JAK2 and CALR were identified. The ASXL1 L731Yfs*12, ASXL1 Q1433Q, ASXL1 p.G646Wfs*10 and TET2 Y1245C variants were found to be putative novel variants. Other known variants were identified in $A B L 1, D N M T 3 A, R U N X 1, S F 3 B 1$ and $U 2 A F 1$. The study findings support the notion that the co-occurrence of multiple variants within a single sample results in a potentially synergistic effect that promotes disease development and progression, and contributes towards higher symptom burden, poorer prognosis, higher risk of leukaemic transformation, and drug resistance [19, 37-42]. In $A B L 1$, point mutations that confer resistance against tyrosine kinase inhibitors in patients with $\mathrm{CML}$ have been discovered in more than 50 different hotspots in the kinase domain [43-46]. It is possible that $A B L 1$ N331S identified in this study may confer drug resistance in a similar fashion to those that have previously been identified.

As MPNs are a clonally heterogenous and multifactorial group of diseases, differences in gene dosage and clonal architecture, the order of mutation acquisition and even germline predisposition can contribute towards MPN pathogenesis as well as differences in disease phenotype and prognosis [47, 48]. In addition, other factors such as lineage bias in haematopoietic stem cells, changes in the bone marrow microenvironment, and aging have also been reported [49]. Further studies should aim to experimentally characterise the functional impact of the variants identified and investigate their possible synergistic effects and biological significance in myeloid disorders, especially in MPN. The putative novel variants should be further investigated using in vitro candidate gene approaches via protein function assays and bioinformatics analyses in order to elucidate their impact especially in poorly characterized proteins [50, 51].

In this study, all NGS-identified variants were confirmed via bidirectional Sanger sequencing except for the SNVs JAK2 V617F and U2AF1 Q157P where the allelic frequencies were $<15 \%$, whereas DMNT3A P385P was not confirmed due to nonspecific primer binding during the PCR step (Table 2 , Supplementary Figure S3). Sanger sequencing is widely regarded as the 'gold standard' for the validation of NGS-detected variants as it can discriminate true variants from NGS artifacts or sequencing errors [52]. However, as the Sanger method relies on the detection of fluorescence, it has limited sensitivity when detecting variants with low allele frequencies; particularly in mosaic tumour samples, leading to false negative results [53-55]. The sensitivity of Sanger sequencing has been reported to be around 15-20\% allele frequency, whereas NGS has a sensitivity of approximately $1 \%$ allele frequency [56]. Therefore, best practice standards for NGS variant confirmation should include alternative methods with higher sensitivity, such as droplet digital PCR or allele-specific qPCR, followed by high-resolution melting curve analysis for the confirmation of variants with low allele frequencies [52, 57]. Nevertheless, such methods are also accompanied by technical limitations and caveats which should be addressed and optimised for their specific intended purposes [58].

Future studies may also benefit from the use of an expanded bioinformatics pipeline which will ultimately provide a greater wealth of information to the clinician, such as the identification of clonal haematopoiesis of indeterminate potential (CHIP, which shows evidence of clonal expansion), monitoring of minimal residual disease, determination of disease predisposition, as well as accurate prediction of treatment response/outcome [59,60]. A larger study cohort as well as the collection of samples and clinical data at follow-up as well as data on response to therapy and survival will allow for better genotypephenotype associations and contribute towards the better understanding of MPNs.

\section{Conclusions}

In summary, the performance of this custom NGS panel indicates towards potential clinical utility in genetic profiling of MPN patients. However, further performance optimisation is required especially for regions with poor coverage depth to ensure that the custom NGS panel will serve as a robust and reliable tool for the personalised management of MPNs. It is hoped that the data generated from the screening of MPN patients using the custom NGS panel will also contribute towards the MPN knowledgebase, and support the adoption of more accurate, genomics-based disease classification as well as prognostic frameworks for the management of MPNs.

\section{Abbreviations}

AML Acute Myeloid Leukaemia

bp base pair

CHIP Clonal Haematopoiesis of Indeterminate Potential

CML Chronic Myeloid Leukaemia

CNL Chronic Neutrophilic Leukaemia

COSMIC Catalogue of Somatic Mutations in Cancer

DNA Deoxyribonucleic Acid

dNTP dideoxynucleotide phosphates

ET Essential Thrombocythaemia

ExAC Exome Aggregation Consortium

ESP6500 NHLBI Exome Sequencing Project 
gnomAD Genome Aggregation Database

IGV Integrative Genomics Viewer

MDS Myelodysplastic Syndrome

MDS/MPN Myelodysplastic/Myeloproliferative Neoplasms

MF Myelofibrosis

MAF Minor allele frequency

MPN Myeloproliferative neoplasms

NGS Next-Generation Sequencing

nSNV nonsynonymous Single Nucleotide Variant

Ph Philadelphia

PMF Primary Myelofibrosis

PV Polycythaemia Vera

SNV Single Nucleotide Variant

sSNV synonymous Single Nucleotide Variant

TN Triple-Negative

VAF Variant Allele Frequency

\section{Declarations}

\section{Ethics approval and consent to participate}

The study was conducted in accordance to the guidelines of the Declaration of Helsinki, and as approved by the Sunway Medical Centre Independent Research Ethics Committee (SREC 008/2018/FR, date of approval 27 th June 2018), Universiti Kebangsaan Malaysia Medical Centre Research Ethics Committee (UKM FPR.4/244/FF-2018-420, date of approval 29 th November 2018), Medical Research \& Ethics Committee Ministry of Health Malaysia (MREC 8KKM/NIHSEC/P-19-99, date of approval 22 ${ }^{\text {nd }}$ January 2019), and Sunway University Research Ethics Committee (PGSUREC 2019/010, date of approval $9^{\text {th }}$ April 2019). Informed consent was obtained from all subjects involved in the study.

\section{Consent for publication}

Informed consent was obtained from the funding institution and all subjects involved in the study.

\section{Availability of data and materials}

The datasets generated and/or analysed during the current study are available in the NCBI BioProject repository, PRJNA754686.

\section{Competing interests}

The authors declare that they have no competing interests.

\section{Funding}

This research was funded by the Sunway Medical Centre Research Fund, grant number SRC/F/18/006. The APC was funded by the Sunway Medical Centre Publication Fund.

\section{Authors' contributions}

Conceptualization, C.L.W. and A.V.; methodology, C.L.W., A.V., Y.P.C., and N.Z.A.; software, Y.P.C. and Y.M.P.,; validation, Y.P.C., N.Z.A. and J.T.; formal analysis, J.T. and Y.P.C.; investigation, J.T., Y.P.C. and N.Z.A.; resources, C.L.W., A.V., K.M.C., V.S and Y.M.P.; data curation, J.T., Y.P.C.; writing-original draft preparation, J.T.; writing-review and editing, J.T., Y.P.C., N.Z.A., C.K.M., V.S., N.R.T., Y.M.P., A.V., M.A.L., and C.L.W.; visualization, J.T. and Y.P.C.; supervision, Y.P.C., N.Z.A., C.L.W., A.V. and M.A.L.; project administration, Y.P.C., N.Z.A. and J.T.; funding acquisition, C.L.W. All authors have read and agreed to the published version of the manuscript.

\section{Acknowledgements}


The authors would like to thank Sunway Medical Centre for funding the study.

\section{References}

1. Cervantes F, Passamonti F, Barosi G. Life expectancy and prognostic factors in the classic BCR/ABL-negative myeloproliferative disorders. Leukemia. 2008;22(5):905-14.

2. Vannucchi AM, Barbui T, Cervantes F, Harrison C, Kiladjian JJ, Kroger N, et al. Philadelphia chromosome-negative chronic myeloproliferative neoplasms: ESMO Clinical Practice Guidelines for diagnosis, treatment and follow-up. Annals of oncology : official journal of the European Society for Medical Oncology. 2015;26 Suppl 5:v85-99.

3. Mullally A, Bruedigam C, Poveromo L, Heidel FH, Purdon A, Vu T, et al. Depletion of Jak2V617F myeloproliferative neoplasm-propagating stem cells by interferon-a in a murine model of polycythemia vera. 2013;121(18):3692-702.

4. Swerdlow SH, Campo E, Harris NL, Jaffe E, Pileri S, Stein H, et al. WHO classification of tumours of haematopoietic and lymphoid tissues. Revised 4th edition ed. Lyon: International Agency for Research on Cancer (IARC) Press; 2017.

5. Arber DA, Orazi A, Hasserjian R, Thiele J, Borowitz MJ, Le Beau MM, et al. The 2016 revision to the World Health Organization classification of myeloid neoplasms and acute leukemia. Blood. 2016;127(20):2391-405.

6. Buhr T, Hebeda K, Kaloutsi V, Porwit A, Van der Walt J, Kreipe H. European Bone Marrow Working Group trial on reproducibility of World Health Organization criteria to discriminate essential thrombocythemia from prefibrotic primary myelofibrosis. Haematologica. 2012;97(3):360-5.

7. Wilkins BS, Erber WN, Bareford D, Buck G, Wheatley K, East CL, et al. Bone marrow pathology in essential thrombocythemia: interobserver reliability and utility for identifying disease subtypes. Blood. 2008;111(1):60-70.

8. Brousseau M, Parot-Schinkel E, Moles MP, Boyer F, Hunault M, Rousselet MC. Practical application and clinical impact of the WHO histopathological criteria on bone marrow biopsy for the diagnosis of essential thrombocythemia versus prefibrotic primary myelofibrosis. Histopathology. 2010;56(6):75867.

9. Grinfeld J, Nangalia J, Baxter EJ, Wedge DC, Angelopoulos N, Cantrill R, et al. Classification and Personalized Prognosis in Myeloproliferative Neoplasms. N Engl J Med. 2018;379(15):1416-30.

10. Ye K, Schulz MH, Long Q, Apweiler R, Ning Z. Pindel: a pattern growth approach to detect break points of large deletions and medium sized insertions from paired-end short reads. Bioinformatics. 2009;25(21):2865-71.

11. Landrum MJ, Lee JM, Riley GR, Jang W, Rubinstein WS, Church DM, et al. ClinVar: public archive of relationships among sequence variation and human phenotype. Nucleic Acids Res. 2014;42(Database issue):D980-5.

12. Tate JG, Bamford S, Jubb HC, Sondka Z, Beare DM, Bindal N, et al. COSMIC: the Catalogue Of Somatic Mutations In Cancer. Nucleic Acids Research. 2018;47(D1):D941-D7.

13. Sherry ST, Ward MH, Kholodov M, Baker J, Phan L, Smigielski EM, et al. dbSNP: the NCBI database of genetic variation. Nucleic Acids Res. 2001;29(1):308-11.

14. Yates AD, Achuthan P, Akanni W, Allen J, Allen J, Alvarez-Jarreta J, et al. Ensembl 2020. Nucleic Acids Research. 2019;48(D1):D682-D8.

15. Ye J, Coulouris G, Zaretskaya I, Cutcutache I, Rozen S, Madden TL. Primer-BLAST: a tool to design target-specific primers for polymerase chain reaction. BMC Bioinformatics. 2012;13:134.

16. Waterhouse AM, Procter JB, Martin DMA, Clamp M, Barton GJ. Jalview Version 2-a multiple sequence alignment editor and analysis workbench. Bioinformatics. 2009;25(9):1189-91.

17. Abdel-Wahab O, Kilpivaara O, Patel J, Busque L, Levine RL. The most commonly reported variant in ASXL1 (c.1934dupG;p.Gly646TrpfsX12) is not a somatic alteration. Leukemia. 2010;24(9):1656-7.

18. Nangalia J, Massie CE, Baxter EJ, Nice FL, Gundem G, Wedge DC, et al. Somatic CALR Mutations in Myeloproliferative Neoplasms with Nonmutated JAK2. New England Journal of Medicine. 2013;369(25):2391-405

19. Papaemmanuil E, Gerstung M, Malcovati L, Tauro S, Gundem G, Van Loo P, et al. Clinical and biological implications of driver mutations in myelodysplastic syndromes. Blood. 2013;122(22):3616-27; quiz 99.

20. Grossmann V, Kohlmann A, Eder C, Haferlach C, Kern W, Cross NC, et al. Molecular profiling of chronic myelomonocytic leukemia reveals diverse mutations in $>80 \%$ of patients with TET2 and EZH2 being of high prognostic relevance. Leukemia. 2011;25(5):877-9.

21. Pratcorona M, Abbas S, Sanders MA, Koenders JE, Kavelaars FG, Erpelinck-Verschueren CA, et al. Acquired mutations in ASXL1 in acute myeloid leukemia: prevalence and prognostic value. Haematologica. 2012;97(3):388-92.

22. Traina F, Visconte V, Jankowska AM, Makishima H, O'Keefe CL, Elson P, et al. Single nucleotide polymorphism array lesions, TET2, DNMT3A, ASXL1 and CBL mutations are present in systemic mastocytosis. PLoS One. 2012;7(8):e43090.

23. Cui Y, Li B, Jiang Q, Xu Z, Qin T, Zhang P, et al. [CSF3R, ASXL1,SETBP1, JAK2 V617F and CALR mutations in chronic neutrophilic leukemia]. Zhonghua xue ye xue za zhi = Zhonghua xueyexue zazhi. 2014;35(12):1069-73.

24. Ross JS, Gay LM, Wang K, Ali SM, Chumsri S, Elvin JA, et al. Nonamplification ERBB2 genomic alterations in 5605 cases of recurrent and metastatic breast cancer: An emerging opportunity for anti-HER2 targeted therapies. Cancer. 2016;122(17):2654-62.

25. Shain AH, Garrido M, Botton T, Talevich E, Yeh I, Sanborn JZ, et al. Exome sequencing of desmoplastic melanoma identifies recurrent NFKBIE promoter mutations and diverse activating mutations in the MAPK pathway. Nature genetics. 2015;47(10):1194-9. 
26. Yap YS, Singh AP, Lim JHC, Ahn JH, Jung KH, Kim J, et al. Elucidating therapeutic molecular targets in premenopausal Asian women with recurrent breast cancers. NPJ breast cancer. 2018;4:19.

27. Shimoda K, Shide K, Kameda T, Hidaka T, Kubuki Y, Kamiunten A, et al. TET2 Mutation in Adult T-Cell Leukemia/Lymphoma. Journal of clinical and experimental hematopathology : JCEH. 2015;55(3):145-9.

28. Wang J, Ai X, Gale RP, Xu Z, Qin T, Fang L, et al. TET2, ASXL1 and EZH2 mutations in Chinese with myelodysplastic syndromes. Leuk Res. 2013;37(3):30511.

29. Metzeler KH, Maharry K, Radmacher MD, Mrózek K, Margeson D, Becker H, et al. TET2 mutations improve the new European LeukemiaNet risk classification of acute myeloid leukemia: a Cancer and Leukemia Group B study. Journal of clinical oncology : official journal of the American Society of Clinical Oncology. 2011;29(10):1373-81.

30. Ha JS, Jeon DS, Kim JR, Ryoo NH, Suh JS. Analysis of the Ten-Eleven Translocation 2 (TET2) gene mutation in myeloproliferative neoplasms. Annals of clinical and laboratory science. 2014;44(2):173-9.

31. Graubert TA, Shen D, Ding L, Okeyo-Owuor T, Lunn CL, Shao J, et al. Recurrent mutations in the U2AF1 splicing factor in myelodysplastic syndromes. Nature genetics. 2011;44(1):53-7.

32. Yoshida K, Sanada M, Shiraishi Y, Nowak D, Nagata Y, Yamamoto R, et al. Frequent pathway mutations of splicing machinery in myelodysplasia. Nature. 2011;478(7367):64-9.

33. Papaemmanuil E, Cazzola M, Boultwood J, Malcovati L, Vyas P, Bowen D, et al. Somatic SF3B1 mutation in myelodysplasia with ring sideroblasts. N Engl J Med. 2011;365(15):1384-95.

34. Aird D, Ross MG, Chen W-S, Danielsson M, Fennell T, Russ C, et al. Analyzing and minimizing PCR amplification bias in Illumina sequencing libraries. Genome Biology. 2011;12(2):R18.

35. Benjamini Y, Speed TP. Summarizing and correcting the GC content bias in high-throughput sequencing. Nucleic Acids Research. 2012;40(10):e72-e.

36. Pereira R, Oliveira J, Sousa M. Bioinformatics and Computational Tools for Next-Generation Sequencing Analysis in Clinical Genetics. Journal of clinical medicine. 2020;9(1).

37. Haferlach T, Nagata Y, Grossmann V, Okuno Y, Bacher U, Nagae G, et al. Landscape of genetic lesions in 944 patients with myelodysplastic syndromes. Leukemia. 2014;28(2):241-7.

38. Thol F, Kade S, Schlarmann C, Löffeld P, Morgan M, Krauter J, et al. Frequency and prognostic impact of mutations in SRSF2, U2AF1, and ZRSR2 in patients with myelodysplastic syndromes. Blood. 2012;119(15):3578-84.

39. Lindsley RC, Saber W, Mar BG, Redd R, Wang T, Haagenson MD, et al. Prognostic Mutations in Myelodysplastic Syndrome after Stem-Cell Transplantation. N Engl J Med. 2017;376(6):536-47.

40. Alberti MO, Srivatsan SN, Shao J, McNulty SN, Chang GS, Miller CA, et al. Discriminating a common somatic ASXL1 mutation (c.1934dup; p.G646Wfs`12) from artifact in myeloid malignancies using NGS. Leukemia. 2018;32(8):1874-8.

41. Grinfeld J, Nangalia J, Green AR. Molecular determinants of pathogenesis and clinical phenotype in myeloproliferative neoplasms. Haematologica. 2017;102(1):7-17.

42. Papaemmanuil E, Gerstung M, Bullinger L, Gaidzik VI, Paschka P, Roberts ND, et al. Genomic Classification and Prognosis in Acute Myeloid Leukemia. N Engl J Med. 2016;374(23):2209-21.

43. Chandrasekhar C, Kumar PS, Sarma PVGK. Novel mutations in the kinase domain of BCR-ABL gene causing imatinib resistance in chronic myeloid leukemia patients. Scientific Reports. 2019;9(1):2412.

44. Soverini S, Rosti G, lacobucci I, Baccarani M, Martinelli G. Choosing the best second-line tyrosine kinase inhibitor in imatinib-resistant chronic myeloid leukemia patients harboring Bcr-Abl kinase domain mutations: how reliable is the IC四? The oncologist. 2011;16(6):868-76.

45. Wylie AA, Schoepfer J, Jahnke W, Cowan-Jacob SW, Loo A, Furet P, et al. The allosteric inhibitor ABL001 enables dual targeting of BCR-ABL1. Nature. 2017;543(7647):733-7.

46. Klco JM, Vij R, Kreisel FH, Hassan A, Frater JL. Molecular pathology of myeloproliferative neoplasms. American journal of clinical pathology. 2010;133(4):602-15.

47. Ortmann CA, Kent DG, Nangalia J, Silber Y, Wedge DC, Grinfeld J, et al. Effect of mutation order on myeloproliferative neoplasms. N Engl J Med. 2015;372(7):601-12.

48. Nangalia J, Nice FL, Wedge DC, Godfrey AL, Grinfeld J, Thakker C, et al. DNMT3A mutations occur early or late in patients with myeloproliferative neoplasms and mutation order influences phenotype. Haematologica. 2015;100(11):e438-42.

49. Tan J, Chow YP, Abidin NZ, Veerakumarasivam A, Wong CL. From Driver Mutations to Genomic Classification: Current \& Future Perspectives on Myeloproliferative Neoplasms. Malaysian Journal of Medicine and Health Sciences. 2021.

50. Robevska G, van den Bergen JA, Ohnesorg T, Eggers S, Hanna C, Hersmus R, et al. Functional characterization of novel NR5A1 variants reveals multiple complex roles in disorders of sex development. Human mutation. 2018;39(1):124-39.

51. Wheway G, Nazlamova L, Meshad N, Hunt S, Jackson N, Churchill A. A Combined in silico, in vitro and Clinical Approach to Characterize Novel Pathogenic Missense Variants in PRPF31 in Retinitis Pigmentosa. Front Genet. 2019;10:248.

52. Beck TF, Mullikin JC, Biesecker LG. Systematic Evaluation of Sanger Validation of Next-Generation Sequencing Variants. Clinical chemistry. 2016;62(4):647-54.

53. Alghasham N, Alnouri Y, Abalkhail H, Khalil S. Detection of mutations in JAK2 exons 12-15 by Sanger sequencing. Int J Lab Hematol. 2016;38(1):34-41. 
54. Braunholz D, Obieglo C, Parenti I, Pozojevic J, Eckhold J, Reiz B, et al. Hidden mutations in Cornelia de Lange syndrome limitations of sanger sequencing in molecular diagnostics. Human mutation. 2015;36(1):26-9.

55. Paparini A, Gofton A, Yang R, White N, Bunce M, Ryan UM. Comparison of Sanger and next generation sequencing performance for genotyping Cryptosporidium isolates at the 18S rRNA and actin loci. Experimental parasitology. 2015;151-152:21-7.

56. Tsiatis AC, Norris-Kirby A, Rich RG, Hafez MJ, Gocke CD, Eshleman JR, et al. Comparison of Sanger sequencing, pyrosequencing, and melting curve analysis for the detection of KRAS mutations: diagnostic and clinical implications. The Journal of molecular diagnostics : JMD. 2010;12(4):425-32.

57. Kuo FC, Mar BG, Lindsley RC, Lindeman NI. The relative utilities of genome-wide, gene panel, and individual gene sequencing in clinical practice. Blood. 2017;130(4):433-9.

58. Słomka M, Sobalska-Kwapis M, Wachulec M, Bartosz G, Strapagiel D. High Resolution Melting (HRM) for High-Throughput Genotyping-Limitations and Caveats in Practical Case Studies. International journal of molecular sciences. 2017;18(11).

59. Teer JK, Zhang Y, Chen L, Welsh EA, Cress WD, Eschrich SA, et al. Evaluating somatic tumor mutation detection without matched normal samples. Human genomics. 2017;11(1):22.

60. Baer C, Walter W, Hutter S, Twardziok S, Meggendorfer M, Kern W, et al. "Somatic" and "pathogenic" - is the classification strategy applicable in times of large-scale sequencing? Haematologica. 2019;104(8):1515-20.

61. Guerra ÁP, Calvo EP, Wasserman M, Chaparro-Olaya J. Producción de proteínas recombinantes de Plasmodium falciparum en Escherichia coli. Biomédica. 2016;36(Sup1):97-108.

\section{Figures}

\begin{tabular}{|c|c|c|c|c|c|c|c|c|c|c|c|c|c|}
\hline \multirow[b]{3}{*}{ Gene } & \multirow[b]{3}{*}{ Protein Change } & \multicolumn{6}{|c|}{ Run 1} & \multicolumn{6}{|c|}{ Run 2} \\
\hline & & \multirow[b]{2}{*}{ 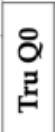 } & \multirow[b]{2}{*}{$\begin{array}{l}\vec{a} \\
z \\
\vec{n}\end{array}$} & \multicolumn{4}{|c|}{\begin{tabular}{|l|l|} 
Tru Q7 & Seraseq \\
\end{tabular}} & \multirow[b]{2}{*}{$\begin{array}{l}\text { वे } \\
\text { 己ే } \\
\end{array}$} & \multirow[b]{2}{*}{$\begin{array}{l}\tilde{a} \\
\text { ż } \\
k\end{array}$} & \multicolumn{2}{|c|}{ Tru Q7 } & \multicolumn{2}{|c|}{ Seraseq } \\
\hline & & & & 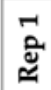 & 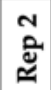 & 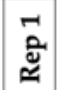 & N & & & 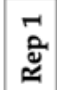 & N & 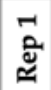 & N \\
\hline ABL1 & p.T315I & & & & & TP & TP & & & & & TP & $\mathrm{TP}$ \\
\hline \multirow[t]{2}{*}{ ASXL1 } & p.E635fs*15 & & & & & TP & TP & & & & & TP & TP \\
\hline & p.G646fs*12 & FP & & & & TP & TP & FP & & & & TP & TP \\
\hline CALR & p.L367fs*46 & & & & & TP & TP & & & & & TP & TP \\
\hline \multirow[t]{2}{*}{ CBL } & p.R420Q & & & & & TP & TP & & & & & TP & TP \\
\hline & p.L380P & & & & & TP & TP & & & & & TP & TP \\
\hline \multirow[t]{2}{*}{ CEBPA } & p.H24fs*84 & & & & & TP & TP & & & & & TP & TP \\
\hline & p.K313_V314insK & & & & & TP & TP & & & & & TP & TP \\
\hline CSF3R & p.T618I & & & & & TP & TP & & & & & TP & TP \\
\hline \multirow[t]{4}{*}{ FLT3 } & c.1759_1800dup & & & & & TP & TP & & & & & TP & TP \\
\hline & dup chr13:28,608,250-28,608,277 & & & & & TP & TP & & & & & TP & TP \\
\hline & p.D835Y & & & TP & TP & TP & TP & & & TP & TP & TP & TP \\
\hline & p. $\Delta \mathrm{I} 836$ & & & TP & TP & & & & & TP & TP & & \\
\hline IDH1 & p.R132C/H & & TP & TP & TP & TP & TP & & TP & TP & TP & TP & TP \\
\hline \multirow[t]{2}{*}{$\mathrm{IDH} 2$} & p.R140Q & & & TP & $\mathrm{TP}$ & & & & & TP & TP & & \\
\hline & p.R172K & & & TP & TP & & & & & TP & TP & & \\
\hline \multirow[t]{2}{*}{$J A K 2$} & p.V617F & & TP & TP & TP & TP & TP & & TP & TP & TP & TP & TP \\
\hline & p.N542_E543del & & & & & TP & TP & & & & & TP & TP \\
\hline KIT & p.D816V & & & TP & TP & & & & & TP & TP & & \\
\hline$M P L$ & p.W515L & & & & & TP & TP & & & & & FN & TP \\
\hline NPM1 & p.W288fs ${ }^{*} 12$ & & & & & TP & TP & & & & & TP & TP \\
\hline PDGFRA & p.D842V & & & TP & TP & & & & & TP & TP & & \\
\hline \multirow[t]{2}{*}{ SF3B1 } & p.K700E & & & & & TP & TP & & & & & TP & TP \\
\hline & p.K666N & & & & & TP & TP & & & & & TP & TP \\
\hline SRSF2 & p.P95_R102del & & & & & TP & TP & & & & & TP & TP \\
\hline U2AF1 & p.S34F & & & & & TP & TP & & & & & TP & TP \\
\hline
\end{tabular}

\section{Figure 1}

Combined analysis of variants with the DNA Amplicon and Pindel apps to evaluate the performance of the custom 22-gene NGS panel based on two identical but independent NGS runs using reference standards. The MPL W515L variant in the Seraseq Myeloid Mutation Mix (Seraseq) was not detected in one of the replicates, giving the custom NGS panel a sensitivity of $99.2 \%$. One variant was detected in the wild-type reference standard TruQ0, giving the panel a specificity of $96.3 \%$. The custom NGS panel also had a positive predictive value of $97.7 \%$, an average intra-run and inter-run concordance of $98.8 \%$ [range 95.2\%-100\%] and 99.0\% [range 95.2\%-100\%] respectively, and was able to detect variants at as low as $1 \%$ allele frequency. FP, False positive; TP, True positive; FN; False negative; Rep 1, Replicate 1; Rep 2, Replicate 2. 


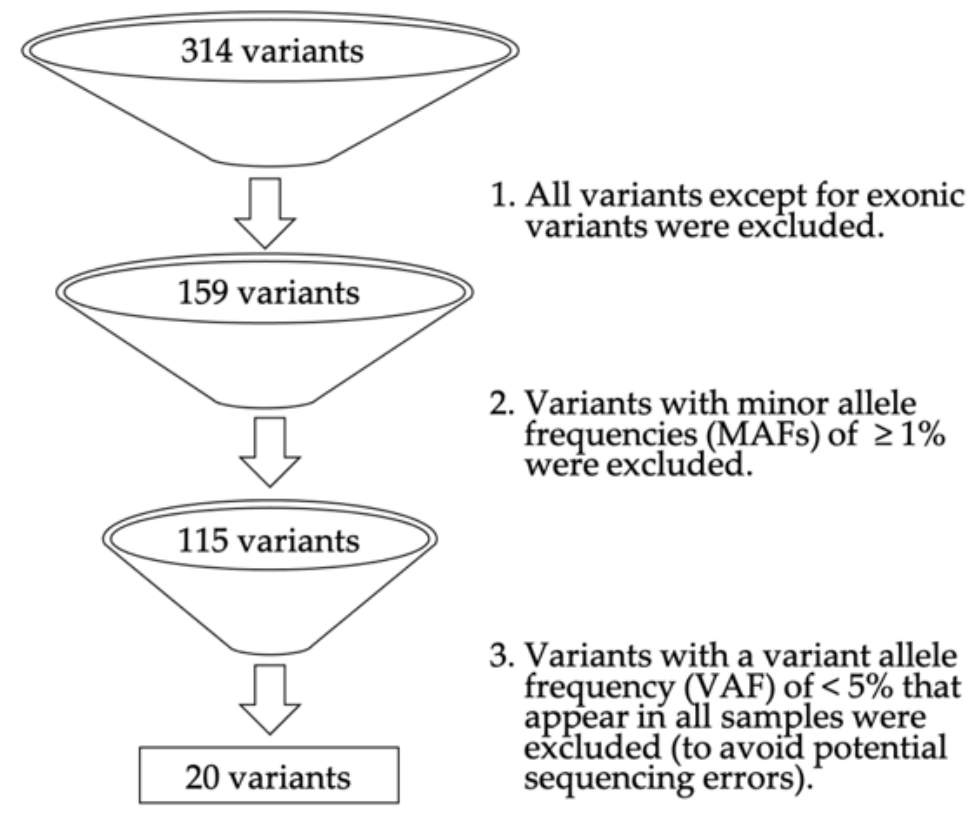

Figure 2

Variant filtering and prioritization process.

\section{Supplementary Files}

This is a list of supplementary files associated with this preprint. Click to download.

- AdditionalFile1AnalysisofGeneticVariantsinMyeloproliferativeNeoplasmsUsinga22GeneNextGenerationSequencingPanel.xlsx

- SupplementaryMaterialAnalysisofGeneticVariantsinMyeloproliferativeNeoplasmsUsinga22GeneNextGenerationSequencingPanel.docx 